\title{
Zur Psychoanalyse des Alterns und Sterbens
}

\section{Timo Storck}

(C) Springer Medizin Verlag GmbH, ein Teil von Springer Nature 2019

Der Animationsfilm Coco - Lebendiger als das Leben! (USA, 2017, Regisseur L. Unkrich, Produktion Walt Disney Pictures/Pixar Animation Studios) thematisiert den mexikanischen Día de Muertos, den Tag der Toten (von der UNESCO als immaterielles Kultur-Erbe der Menschheit anerkannt). Der 12-jährige Protagonist Miguel folgt seiner Leidenschaft für die Musik und gerät auf der Suche nach der Identität seines verschwundenen Ururgroßvaters ins Reich der Toten. In der Erzählung ist es den Verstorbenen an einem Tag im Jahr möglich, ihre lebenden Verwandten zu besuchen: sofern diese am Tag der Toten ein Foto von ihnen aufstellen. Bei wem das nicht der Fall ist, der darf die Ausgangskontrolle aus dem Reich der Toten nicht passieren. Dort wiederum existieren die Toten nur so lange, wie jemand unter den Lebenden sich an sie erinnern kann. Ein Teil der Geschichte besteht nun darin, dass Miguel dafür zu sorgen versucht, dass a) sein Ururgroßvater Hector von seiner noch lebenden Tochter Coco, Miguels Urgroßmutter, nicht vergessen und dass b) dessen Bild in liebevoller Erinnerung aufgestellt wird.

Der Film thematisiert ästhetisch und narrativ in beeindruckender und bewegender Weise die Auseinandersetzung mit Entwicklung, Erinnerung und Endlichkeit. Es geht darum, wie die Toten in der Erinnerung weiterleben (und um die Grenzen dessen, wie eine emotionale Verbindung über das Ende des Lebens hinaus bestehen bleiben kann), aber auch um das Ende des Lebens (in Form der Figur der Coco, die am Ende im Anschluss an ein Wiedererwecken ihrer Lebenserinnerungen aus dem Leben scheiden kann).

Diese Einführung in das Schwerpunktthema „Altern und Sterben“ setzt sich, wie die folgenden Beiträge, mit einem Teilbereich des Themas auseinander (für eine geistesgeschichtliche Verortung der Bedeutung des Todes für die Psychotherapie:

Prof. Dr. T. Storck (凹)

Psychologische Hochschule Berlin, Am Köllnischen Park 2, 10179 Berlin, Deutschland

E-Mail: t.storck@psychologische-hochschule.de 
Vogel 2012). Es geht im Weiteren um Altern, Sterben und Tod unter der Perspektive der Endlichkeit und der Auseinandersetzung damit aus psychoanalytischer Sicht. Aus der Freud'schen bzw. psychoanalytischen „Thanatologie“ (Eissler 1978 [1955]) wird mit dem Aspekt des Todes als Erlebnisinhalt ein wesentlicher Teil herausgegriffen; dem gegenüber steht der Aspekt eines triebhaften oder sonstigen im Menschen wirkenden Motivs im Hintergrund. Allerdings bedarf der leicht dahingesagte Ausdruck „Tod als Erlebnisinhalt“ einiger kurzer Erläuterungen: Freud vertritt den konzeptuellen Standpunkt der eigenen Unsterblichkeit im Unbewussten. Auch kann zu Recht eingewandt werden, dass wir vom (eigenen) Tod nichts wissen können, eine Erlebnisperspektive steht uns schlicht nicht offen (der Tod kann nicht erlebt werden), noch nicht einmal thanatoempathisch, denn anders als im Film Coco können wir uns nicht mit Verstorbenen über das Leben nach dem Tod austauschen. Allerdings werden Tod oder Endlichkeit sehr wohl Gegenstand unseres inneren Erlebens, wenn wir uns damit beschäftigen, Fantasien dazu nachgehen oder etwas dazu fühlen - dies wird in einer „Psychologie des Todes“ (Eissler 1978 [1955]) betrachtet.

Coco ist als Einzug des Todes in die ,familienfreundliche“ Unterhaltungskultur zu sehen (immerhin ohne Altersfreigabe-Beschränkung!). Dabei ging es dem Familienkino in der Umgehung des ,realen Todes“ (Hinze 2008) lange Zeit wie der Psychoanalyse, der es traditionell offenkundig mehr um den „kleinen Tod“ als um den großen gegangen ist, mehr um (tabuisierte) Sexualität als um Endlichkeit - der Tod war als Thema nicht gern gesehen, auch wenn er als ,fact of life“"immer mal wieder benannt worden ist (Money-Kyrle 1971). Freud hat sich bekanntermaßen persönlich eher übermäßig mit dem eigenen Tod beschäftigt, und die Realität des Todes beherrschte ,,zunehmend auch sein persönliches Dasein mit kaum vorstellbarer Unbarmherzigkeit“" (Whitebook 2017, S. 345). Hinsichtlich der Theoriebildung allerdings hat - bezeichnenderweise? - unter den Dramen Sophokles' Ödipus Rex eindeutig mehr Aufmerksamkeit auf sich gezogen als Ödipus auf Kolonos, und noch randständiger ist Antigone zu verorten (wenn auch Lacans Konzeption des ,zweiten Todes“ herauszustellen ist; Lacan 1996 [1959/60]).

Zwar thematisiert Freud (1920g) ab 1920 beständig den Todestrieb, bei dem es, biologische Bezüge eingeklammert, um die Kraft einer Entbindung oder Destruktivität geht, die dem erotisch-bindenden Moment entgegensteht, doch bleibt die Konzeption der Sterblichkeit als Element des Psychischen allenfalls angedeutet. Freud (1915b, S. 341) schreibt in Zeitgemäßes über Krieg und Tod (ein Text, der auf einem Vortrag mit dem Titel „Wir und der Tod“ beruht; Freud 1915i): „Im Unbewußten sei [sic] jeder von uns von seiner Unsterblichkeit überzeugt“. Er geht von einer (gattungsgeschichtlich eingebetteten) Verleugnung des eigenen Todes aus (wobei der Krieg an dieser rüttele). Denn: „Unser Unbewußtes glaubt nicht an den eigenen Tod, es gebärdet sich wie unsterblich“ (Freud 1915b, S. 350). Anders als etwa für Melanie Klein ist die Todesangst für Freud hier „etwas Sekundäres“, das er sich v. a. als Erwartung von Strafe vorstellt (und so ist die Verbindung zwischen Todesund Kastrationsangst zu verstehen, auf die er wiederholt Bezug nimmt), nicht als ein eigenes Motiv, das die Auseinandersetzung mit Sterblichkeit oder Vergänglichkeit ausdrückte. Durch Freuds Argumentation zieht sich durch, dass der eigene Tod eine besondere Bedeutung im psychischen Erleben haben muss: Wir werden zwar (wenn man so will: eher abstrakt) an die Tatsache des Todes erinnert, im Krieg oder 
durch den Verlust eines nahestehenden Menschen. Das Unbewusste ist ,gegen die Vorstellung des eigenen Todes“ allerdings „unzugänglich“ (Freud 1915b, S. 354). Erst einige Jahre später, mit Einführung der Todestrieb-Konzeption, betrachtet Freud den Tod als etwas, das mit einem eigenständigen Prinzip zu tun hat: „Das Ziel allen Lebens ist der Tod“", schreibt er nun (Freud 1920g, S. 40).

Im weiteren Verlauf der psychoanalytischen Theorieentwicklung finden wir den Tod (Todestrieb) in Konzeptionen der kleinianischen Richtung und dies zwar an prominenter Stelle (als Entwicklungsmotor, in Allianz mit Angst, Aggression und unbewusster Fantasie), jedoch selten selbst als Element der bewussten und unbewussten Vorstellungswelt. Als wegweisende Arbeiten sind Der sterbende Patient von Eissler (1978 [1955]) aus dem Jahr 1955 und einzelne Arbeiten de M'Uzans zu nennen (gesammelt in de M'Uzan 2014), der sich mit der libidinösen Dynamik des Lebensendes auseinandersetzt und hier das „Phänomen einer Libidoexpansion“ im Zuge der Trauerarbeit beschreibt (M’Uzan 1996, S. 227), als „letzte[.] Beziehungserfahrung“ oder ,letzte Dyade“ in Form einer „Arbeit am Übergang“ (M'Uzan 1977, S. 77). In jüngerer Vergangenheit haben Arbeiten zum Altern (und zum Teil zur Alternspsychotherapie) eine breit geführte Auseinandersetzung mit der ,leeren Couch“ (Junkers 2013; Quinodoz 2012 [2008]), also mit der Begrenztheit der Arbeit als Analytiker angestoßen. Wie das Familienkino hat mittlerweile auch die Psychoanalyse erkannt, wie viele drängende Fragen sich an das Thema Altern und Sterben anheften (besonders Grieser 2018).

Diesen widmen sich die Beiträge des vorliegenden Schwerpunkthefts. Zunächst legt Jürgen Grieser etwas vor, das man als „Entwicklungspsychologie des Todes“ bezeichnen kann, wenn er die psychische Beschäftigung mit dem Tod über die verschiedenen Lebensalter beleuchtet. Das führt ihn zur Diskussion verschiedener Weisen der Beschäftigung mit der Sterblichkeit - unter der Perspektive der Triangulierung, des Verhältnisses von Libido und Narzissmus und der Transzendenz. Gabriele Junkers widmet sich allgemeinen Fragen zum Altern und hebt dabei die Bedeutung des Körpers hervor. Sie kann zeigen, dass das Verhältnis zwischen Selbst und Körper im Alter eine Krise erfährt. Der alternde Körper/Leib meldet sich vermehrt als biologischer Körper - in Krankheit, Begrenzungen, limitierten Möglichkeiten und Funktionen. Es bedarf einer Wiederaneignung der eigenen Leiblichkeit im Alter statt einer Spaltung in „fit im Kopf“ auf der einen und eine altersbedingte „somatische Werkzeugstörung" auf der anderen Seite. Jakob Müller und Cécile Loetz legen ein Modell psychoanalytisch orientierter Sterbebegleitung vor und widmen sich, basierend auf den Ergebnissen einer Studie zur Bindungsrepräsentation in der Palliativarbeit, der Paradoxie von Trost oder Hoffnung in der Gesprächsführung mit Sterbenden: Was ist tröstlich, ohne zu ,vertrösten“"? Welche Hoffnung kann die analytische Arbeit in Aussicht stellen, wenn auf eine Weise der Ausgang der Behandlung und des Lebens fest- und bevorsteht? Schließlich diskutiert Michael Ermann die Rolle des Alters für den Psychoanalytiker und entwirft Gedanken zu einer ,psychoanalytischen Altersidentität“", in der das Anerkennen von Grenzen, der Blick auf das Erreichte und das Betrauern des Vergangenen oder Nicht-Erreichten eine Rolle spielen. Steht auf der einen Seite die Gefahr unerkannter Eigenübertragungen, so ist auf der anderen das Ziel einer „Altersweisheit“ zu sehen, deren Kern 
darin besteht, neben einem Reichtum an Erfahrung auch die Tatsache von Grenzen und der eigenen Endlichkeit anzuerkennen.

Interessenkonflikt T. Storck gibt an, dass kein Interessenkonflikt besteht.

\section{Literatur}

Eissler KR (1978) Der sterbende Patient. Zur Psychologie des Todes. frommann-holzboog, Stuttgart Freud S (1915b) Zeitgemäßes über Krieg und Tod. GW X, S 323-355

Freud S (1915i) Wir und der Tod. Psyche - Z Psychoanal 45:132-142

Freud S (1920g) Jenseits des Lustprinzips. GW XIII, S 1-69

Grieser J (2018) Der Tod und das Leben. Vergänglichkeit als Chance zur Entwicklung von Lebendigkeit. Psychosozial, Gießen

Hinze E (2008) Sterben, Endlichkeit und Tod. Psychother Alter 5(2)

Junkers G (Hrsg) (2013) Die leere Couch. Der Abschied von der Arbeit als Psychoanalytiker. Psychosozial, Gießen

Lacan J (1996) Das Seminar. Buch VII. Die Ethik der Psychoanalyse. Quadriga, Weinheim, Berlin

Money-Kyrle R (1971) The aim of psychoanalysis. Int J Psychoanal 52:103-106

de M'Uzan M (1977) Die Arbeit am Übergang. In: de M'Uzan M (Hrsg) Depersonalisation und Kreativität. Psychosozial, Gießen, S 75-91

de M’Uzan M (1996) Der Tod gesteht nie. In: de M’Uzan M (Hrsg), Identität und Tod. Psychosozial, Gießen, S 223-239

de M'Uzan M (2014) Identität und Tod. Psychosozial, Gießen

Quinodoz D (2012) Älterwerden. Eine Entdeckungsreise, 2. Aufl. Psychosozial, Gießen

Vogel RT (2012) Todesthemen in der Psychotherapie. Kohlhammer, Stuttgart

Whitebook J (2017) Freud: Sein Leben und Denken. Klett-Cotta, Stuttgart

Prof. Dr. Timo Storck Diplom-Psychologe, psychologischer Psychotherapeut und Psychoanalytiker (DPV/DGPT/IPA), Professor für klinische Psychologie und Psychotherapie an der Psychologischen Hochschule Berlin. Forschungsschwerpunkte: psychoanalytische Psychosomatik, Theorie und Praxis der Fallbesprechung in der stationären Psychotherapie, psychoanalytische Grundkonzepte, Verstehen in der Psychotherapie, Filmpsychoanalyse, konzeptvergleichende Psychotherapieforschung. 